\title{
Influence of Positron Emitters on Standard $\gamma$-Camera Imaging
}

\author{
Lars Jødal $^{1}$, Pia Afzelius ${ }^{1}$, and Svend Borup Jensen ${ }^{1,2}$ \\ ${ }^{1}$ Department of Nuclear Medicine, Aalborg University Hospital, Aalborg, Denmark; and ${ }^{2}$ Department of Chemistry and Biochemistry, \\ Aalborg University, Aalborg, Denmark
}

Combined PET and SPECT scanning can give supplementary information. However, activity from PET radionuclides can cause background counts and increased dead time in $\gamma$ camera imaging (SPECT or planar) because the 511-keV photons can penetrate collimators designed for lower energies. This study investigated how to manage this issue, including what levels of PET radionuclides can be tolerated when a $\gamma$-camera investigation is performed. Methods: Different combinations of ${ }^{68} \mathrm{Ga}$ (PET radionuclide), ${ }^{99 m T c}$ (low-energy radionuclide), and ${ }^{111} \mathrm{In}$ (medium-energy radionuclide) were scanned by a $\gamma$ camera. Standard low-, medium-, and high-energy collimators were used with the $\gamma$ camera. Dead time and counts near and distant from the sources were recorded. Results: Down scatter from $511 \mathrm{keV}$ can give rise to a considerable number of counts within the ${ }^{99 \mathrm{mTC}}$ or ${ }^{111}$ In energy windows, especially when the PET source is close to the camera head. Over the full camera head, the PET source can result in more counts per megabecquerel than the SPECT source $\left({ }^{99 \mathrm{mTC}}\right.$ or $\left.{ }^{111} \mathrm{In}\right)$. Counts from the PET source were distributed over a large region of the camera head. With medium- and high-energy collimators, the sensitivity to the PET radionuclide was found to be about $10 \%$ of the sensitivity to $99 \mathrm{mTc}$ and about $20 \%$ of the sensitivity to ${ }^{111} \mathrm{In}$, as measured within a $3-\mathrm{cm}$-radius region of interest. Conclusion: If PET radionuclides of activity 1 $\mathrm{MBq}$ or higher are present in the patient at the time of SPECT, a medium-energy collimator should be used. Counts from PET sources will in SPECT usually be seen as a diffuse background rather than as foci. The thick septa of high-energy collimators may result in structure in the image, and a high-energy collimator is recommended only if PET activity is greater than $10 \mathrm{MBq}$.

Key Words: 511 keV; downscatter; dead-time; PET; SPECT; dual-isotope; dual tracer; dual modality

J Nucl Med Technol 2014; 42:42-50

DOI: $10.2967 /$ jnmt.113.131003

$\mathbf{F}$ or molecular imaging, PET and SPECT are often used as alternative techniques, as reflected by the title of a review covering both techniques: "PET versus SPECT..." (1). However, rather than being alternatives, PET and SPECT can supplement each other by allowing the same patient or

Received Aug. 13, 2013; revision accepted Nov. 22, 2013.

For correspondence or reprints contact: Lars Jødal, Department of Nuclear Medicine, Aalborg University Hospital, Hobrovej 18-22, DK-9000 Aalborg, Denmark.

E-mail: lajo@rn.dk

Published online Jan. 27, 2014.

COPYRIGHT (C 2014 by the Society of Nuclear Medicine and Molecular Imaging, Inc. animal to be imaged with more than one tracer. For instance, a patient suspected of having bone infections may be imaged with a combination of ${ }^{99 \mathrm{~m} T c-m e t h y l e n e ~ d i p h o s-~}$ phonate for bone metabolism and ${ }^{18} \mathrm{~F}-\mathrm{FDG}$ as a marker of infection. Or a new PET or SPECT tracer may be tested against an existing gold standard involving the other modality. The potential of dual-tracer PET and SPECT imaging was summarized in a recent perspective by El Fakhri, who asked if the SPECT/PET combination is "ready for prime time." (2)

On the physics level, all radionuclides used in PET emit positrons, resulting in coincident photons of $511-\mathrm{keV}$ energy. On the other hand, radionuclides used in $\gamma$-camera imaging (both SPECT and planar imaging) are not fixed to a single energy, allowing for dual-isotope imaging in which radionuclides are distinguished by the energy of their photons. For example, leukocytes labeled with ${ }^{111}$ In (photon energies of 171 and $245 \mathrm{keV}$ ) can be imaged simultaneously with tracers for bone or bone marrow labeled with ${ }^{99 \mathrm{~m}} \mathrm{Tc}$ (photon energy of $140 \mathrm{keV}$ ) $(3,4)$.

For the sake of brevity, in the remainder of this article the term SPECT will be used for $\gamma$-camera imaging, whether the imaging is tomographic or planar. The phrases SPECT radionuclide and $P E T$ radionuclide will be used to denote radionuclides used in SPECT (e.g., ${ }^{99 \mathrm{~m} T c}$ or ${ }^{111} \mathrm{In}$ ) and PET (e.g., ${ }^{18} \mathrm{~F}$ or ${ }^{68} \mathrm{Ga}$ ), respectively.

Combining PET tracers and SPECT tracers will increase the number of interesting tracer pairs $(2,5)$. However, the 511-keV photons from PET radionuclides can penetrate the collimators of the $\gamma$ camera, resulting in increased dead time and counts not originating from the SPECT tracer. In a recent preclinical study, Chapman et al. (5) used a combined PET/ SPECT/CT imaging system on ${ }^{18} \mathrm{~F}$ and ${ }^{99 \mathrm{~m} T c}$ tracers. PET rejects noncoincident photons and photons of lower energy, and the study found no influence of ${ }^{99} \mathrm{~m} \mathrm{Tc}$ on the PET scan even though the activity of ${ }^{99 \mathrm{~m}} \mathrm{Tc}$ was 10 times higher than that of ${ }^{18} \mathrm{~F}$. Thus, regarding the PET scanning, the researchers had to ensure only that the total number of events from SPECT and PET activity did not cause unacceptable dead time. However, the study found significant artifacts in the SPECT images if the PET radionuclide was present. Consequently, the study recommended a sequential protocol, with SPECT being completed before injection of the PET radionuclide.

Although completing SPECT before injection of the PET radionuclide is a good rule of thumb, it is not a universal solution because factors other than physics may make it necessary to inject a PET tracer before completion of the 
SPECT scanning. For example, a cardiac stress test could

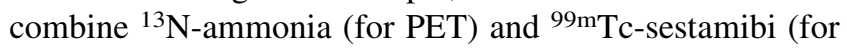
SPECT), injected simultaneously at the time of maximum stress. In this example, because the PET radionuclide decays quickly (half-life $\left[\mathrm{T}_{1 / 2}\right]=10 \mathrm{~min}$ for ${ }^{13} \mathrm{~N}$ ), the SPECT scanning may be performed when sufficient decay of the PET radionuclide has occurred. However, this leaves open the question of how long after the PET injection to wait before performing SPECT.

The aim of the present study was to investigate what amount of PET radionuclides can be tolerated by a standard $\gamma$ camera equipped with either low-, medium-, or high-energy collimators. The investigation covered ${ }^{99} \mathrm{~m} T \mathrm{Tc}$ imaging (low-energy radionuclide) and ${ }^{111} \mathrm{In}$ imaging (medium-energy radionuclide). Given such numbers and the half-life of the relevant PET radionuclide, the clinician or researcher can then estimate when SPECT can be performed.

\section{MATERIALS AND METHODS}

All imaging studies were performed on 2 identical Symbia T16 SPECT/CT scanners (Siemens) equipped with standard $0.95-\mathrm{cm}$ (3/8-in) NaI crystals. The CT part of the scanners was not used in this study. The acquisitions were made with collimators designed for low-energy, high-resolution (LEHR); medium-energy, lowpenetration (MELP); or high-energy (HE) imaging (Table 1). An ultra-high-energy (UHE) collimator designed for 511-keV SPECT was not available at our center but is included in Table 1 for comparison.

Because of practical constraints, the data using the LEHR and MELP collimators were acquired on one camera, whereas the data using the HE collimator were acquired on the other camera.

\section{Comparison of Collimators}

To compare the collimator designs in relation to PET radionuclides, a measure of septal penetration was calculated as follows.

On the basis of the geometry shown in Figure 1, a $\gamma$ ray passing a septum in a collimator will have a minimum path length $p$, which can approximately be expressed as $(6,7)$

$$
p=\frac{s L}{2 D+s}
$$

where $s$ is the septal thickness, $L$ is the hole length, and $D$ is the "diameter" of the holes, that is, the distance between 2 parallel sides (Fig. 1). Equation 1 calculates vertical distance in the septum rather than $p$, but this is a good approximation when holes are much longer than their diameter.

Septal penetration was then calculated as the percentage of 511$\mathrm{keV}$ photons that passed a length $p$ of lead without interactions (i.e., neither absorption nor scattering):

$$
\text { Septal penetration }=e^{-\mu p} \times 100 \% \text {, }
$$

Eq. 2

where

$$
\mu=\frac{\ln 2}{4.1 \mathrm{~mm}}=0.169 \mathrm{~mm}^{-1},
$$

using a half-length of $4.1 \mathrm{~mm}$ for $511-\mathrm{keV}$ photons in lead.

\section{Sources}

In all measurement series, ${ }^{68} \mathrm{Ga}\left(\mathrm{T}_{1 / 2}=67.7 \mathrm{~min}\right)$ was used as a PET source. For a SPECT source, we used ${ }^{99 \mathrm{~m}} \mathrm{Tc}$ as a typical low-energy radionuclide ( $\gamma$ energy of $140 \mathrm{keV}, \mathrm{T}_{1 / 2}=6.01 \mathrm{~h}$ ) and ${ }^{111}$ In as an example of a medium-energy radionuclide ( $\gamma$ energies of 245 and $171 \mathrm{keV}, \mathrm{T}_{1 / 2}=2.8 \mathrm{~d}$ ). Source activities are given in Table 2.

Because septal penetration was expected, small rather than extended sources were used to easily compare the measured images with the physical positions of the sources. Each source consisted of approximately $1 \mathrm{~mL}$ of liquid in a plastic test tube with a $1-\mathrm{cm}$ inner diameter. The 2 sources were placed in symmetric positions $24 \mathrm{~cm}$ apart (Fig. 2). The sources were placed $10 \mathrm{~cm}$ above the surface of the collimator on the lower camera head.

We considered the possibility of surrounding the sources by a scatter medium, but to avoid the complications of ensuring the same amount of medium above and below the sources, we chose the simpler approach we have described here, supplemented with separate scatter measurements (see section below on measured energy spectrum).

\section{Camera Set-up}

The camera heads were in the Symbia "home" position, where both heads are horizontal and at a reproducible distance, although the collimator-to-collimator distance depends on the thickness of the collimator: $73 \mathrm{~cm}$ for the LEHR collimator, $70 \mathrm{~cm}$ for the MELP collimator, and $66 \mathrm{~cm}$ for the HE collimator.

The sources were placed $10 \mathrm{~cm}$ above the lower camera head with collimator (the "close detector"). Thus, the distance to the "distant detector" (upper camera head with collimator) was about $60 \mathrm{~cm}$ for all collimators used.

\begin{tabular}{|c|c|c|c|c|c|c|c|}
\hline \multirow[b]{2}{*}{ Collimator type } & \multicolumn{3}{|c|}{ Holes } & \multirow{2}{*}{$\begin{array}{c}\text { Septal } \\
\text { thickness (mm) }\end{array}$} & \multirow{2}{*}{$\begin{array}{c}\text { Geometric resolution } \\
\text { at } 10 \mathrm{~cm}(\mathrm{~mm})\end{array}$} & \multirow{2}{*}{$\begin{array}{l}p \text { in } \mathrm{mm} \\
(\text { Eq. } 1)^{\dagger}\end{array}$} & \multirow{2}{*}{$\begin{array}{l}\text { Septal penetration } \\
\text { at } 511 \mathrm{keV} \text { (Eq. 2) }{ }^{\dagger}\end{array}$} \\
\hline & Shape & Diameter $(\mathrm{mm})^{\star}$ & Length (mm) & & & & \\
\hline LEHR & Hexagonal & 1.11 & 24.05 & 0.16 & 6.4 & 1.6 & $76 \%$ \\
\hline MELP & Hexagonal & 2.94 & 40.64 & 1.14 & 10.8 & 6.6 & $33 \%$ \\
\hline $\mathrm{HE}$ & Hexagonal & 4 & 59.7 & 2 & 13.2 & 11.9 & $13 \%$ \\
\hline UHE & Hexagonal & 2.5 & 50.5 & 3.4 & 10.6 & 20.4 & $3.2 \%$ \\
\hline
\end{tabular}

TABLE 1

Selected Data from Collimator Specifications (23) 


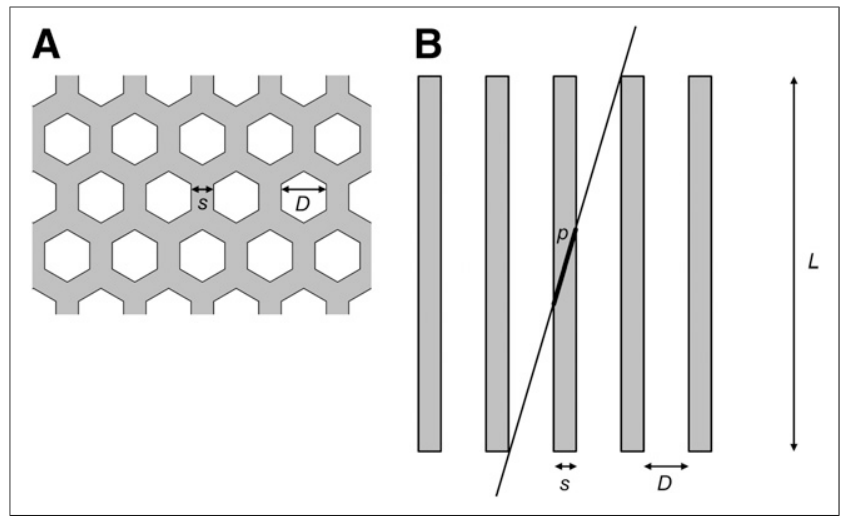

FIGURE 1. Illustration of collimator with hexagonal holes. (A) Top view of collimator: in this example, hole diameter $(D)$ is equal to twice septal thickness (s), as is the case for HE collimator (Table 1). (B) Side view showing y ray penetrating collimator with minimum path length $(p)$ in septa. Hole length $(L)$ is not drawn to scale.

Imaging was performed using a $128 \times 128$ matrix with a $4.8 \times$ $4.8 \mathrm{~mm}$ pixel size. Usual energy windows for SPECT imaging were used: for ${ }^{99 \mathrm{~m}} \mathrm{Tc}$, the energy window was $140 \mathrm{keV} \pm 7.5 \%$ (i.e., a $15 \%$ width), and for ${ }^{111} \mathrm{In}$, the energy windows were 171 $\mathrm{keV} \pm 7.5 \%$ and $245 \mathrm{keV} \pm 7.5 \%$. No energy window at 511 $\mathrm{keV}$ was used because the intention was not to perform 511$\mathrm{keV}$ SPECT but to measure the influence on the energy windows normally used for SPECT for low- and medium-energy radionuclides.

\section{Measured Energy Spectrum from PET Source}

For each collimator, an energy spectrum was recorded with 1-2 $\mathrm{MBq}$ of the PET source, positioned as described above. These recordings were acquired over a time corresponding to $1 \mathrm{MBq}$ in $1 \mathrm{~min}$ (LEHR collimator) or $1 \mathrm{MBq}$ in $10 \mathrm{~min}$ (MELP and HE collimators).

To evaluate the influence of a scatter medium, supplementary recordings were made in which a PET source or a ${ }^{99 \mathrm{~m}} \mathrm{Tc}$ source was placed $11 \mathrm{~cm}$ above the center position of the close detector. Below the source was either air (no scatter medium) or a scatter medium consisting of a water-filled clear acrylic phantom of outer dimensions $11 \times 22 \times 27 \mathrm{~cm}$, with the acrylic being $5 \mathrm{~mm}$ thick.

\section{Acquisitions}

To obtain data for a series of different activities of the PET source, we performed dynamic acquisitions over a long time compared with the half-life of ${ }^{68} \mathrm{Ga}$. Each measurement series consisted of the following series of frames, with each frame being 6 min: frames 1-3, background acquisition; frame 4, PET source $\left({ }^{68} \mathrm{Ga}\right)$ alone; frame 5 , SPECT source $\left({ }^{99 \mathrm{~m}} \mathrm{Tc}\right.$ or $\left.{ }^{111} \mathrm{In}\right)$ alone; and frames 6-125 (12 h in total), both PET source and SPECT source. To allow for the change of the sources, the acquisition paused after frames 3,4 , and 5 .

Five measurement series were performed: series A, LEHR collimator, ${ }^{99 \mathrm{~m}} \mathrm{Tc}$ and ${ }^{68} \mathrm{Ga}$; series B, MELP collimator, ${ }^{99 \mathrm{~m}} \mathrm{Tc}$ and ${ }^{68} \mathrm{Ga}$; series $\mathrm{C}$, HE collimator, ${ }^{99 \mathrm{~m}} \mathrm{Tc}$ and ${ }^{68} \mathrm{Ga}$; series D, MELP collimator, ${ }^{111} \mathrm{In}$ and ${ }^{68} \mathrm{Ga}$; and series E, HE collimator, ${ }^{111} \mathrm{In}$ and ${ }^{68} \mathrm{Ga}$. Because ${ }^{111} \mathrm{In}$ is a medium-energy radionuclide, it was not measured with the LEHR collimator.

\section{Regions of Interest (ROIs)}

ROIs were drawn in the position of both the SPECT and the PET sources. Circular ROIs with a size of 30 pixels $\left(6.9 \mathrm{~cm}^{2}\right)$ were used, which corresponded to a circle with a $3.0-\mathrm{cm}$ diameter, the approximate full width at half maximum of the ${ }^{99 \mathrm{~m}} \mathrm{Tc}$ source as seen on the distant detector with the LEHR collimator. Illustrations of these ROIs are presented in the "Results" section.

\section{Counting Rates and Detector Sensitivities}

For a given area (the full detector or an ROI), the counting rates (counts per second) were calculated as follows:

- Background: total counts in frames 1-3 divided by 1,080 s (3 $\times 6$ min)

- PET source: total counts in frame 4 divided by $360 \mathrm{~s} \mathrm{(6} \mathrm{min)}$

- SPECT source: total counts in frame 5 divided by $360 \mathrm{~s}$ (6 min)

No correction for dead time was made.

The sensitivity (cps/MBq) of a given area for a given source was calculated as follows:

Sensitivity $=($ count rate - background count rate $) /$ activity, Eq. 4

TABLE 2

Collimators, Radionuclides, and Activities Used, Along with Dead Time

\begin{tabular}{|c|c|c|c|c|c|}
\hline \multirow[b]{2}{*}{ Measurement series } & \multirow[b]{2}{*}{ Collimator } & \multirow[b]{2}{*}{ Radionuclide } & \multirow[b]{2}{*}{ Activity (MBq) } & \multicolumn{2}{|c|}{ Dead time } \\
\hline & & & & $\begin{array}{l}\text { Close detector } \\
\text { (10-cm distance) }\end{array}$ & $\begin{array}{l}\text { Distant detector } \\
\text { ( 60-cm distance) }\end{array}$ \\
\hline A & $\begin{array}{l}\text { LEHR } \\
\text { LEHR }\end{array}$ & $\begin{array}{l}{ }^{68} \mathrm{Ga} \\
99 \mathrm{mTc}\end{array}$ & $\begin{array}{l}3.5 \\
9.4\end{array}$ & $\begin{array}{c}21 \% \\
\text { Negligible }\end{array}$ & $\begin{array}{c}4 \% \\
\text { Negligible }\end{array}$ \\
\hline B & $\begin{array}{l}\text { MELP } \\
\text { MELP }\end{array}$ & $\begin{array}{l}{ }^{68} \mathrm{Ga} \\
99 \mathrm{mTc}\end{array}$ & $\begin{array}{r}10.9 \\
9.1\end{array}$ & $\begin{array}{c}5 \% \\
\text { Negligible }\end{array}$ & $\begin{array}{c}1.5 \% \\
\text { Negligible }\end{array}$ \\
\hline $\mathrm{C}$ & $\begin{array}{l}\mathrm{HE} \\
\mathrm{HE}\end{array}$ & $\begin{array}{l}{ }^{68} \mathrm{Ga} \\
99 \mathrm{~m} \mathrm{Tc}\end{array}$ & $\begin{array}{l}6.6 \\
8.2\end{array}$ & $\begin{array}{c}<1 \% \\
\text { Negligible }\end{array}$ & $\begin{array}{l}\text { Negligible } \\
\text { Negligible }\end{array}$ \\
\hline $\mathrm{D}$ & $\begin{array}{l}\text { MELP } \\
\text { MELP }\end{array}$ & $\begin{array}{l}{ }^{68} \mathrm{Ga} \\
111 / \mathrm{l}\end{array}$ & $\begin{array}{l}7.4 \\
9.2\end{array}$ & $\begin{array}{c}3 \% \\
\text { Negligible }\end{array}$ & $\begin{array}{c}<1 \% \\
\text { Negligible }\end{array}$ \\
\hline$E$ & $\begin{array}{l}\text { HE } \\
\mathrm{HE}\end{array}$ & $\begin{array}{l}{ }^{68} \mathrm{Ga} \\
111 \mathrm{In}\end{array}$ & $\begin{array}{l}7.5 \\
9.2\end{array}$ & $\begin{array}{c}<1 \% \\
\text { Negligible }\end{array}$ & $\begin{array}{l}\text { Negligible } \\
\text { Negligible }\end{array}$ \\
\hline
\end{tabular}

Activities are given at time halfway into frame 4 for PET radionuclide $\left({ }^{68} \mathrm{Ga}\right)$ and halfway into frame 5 for SPECT radionuclides $\left({ }^{99 m} \mathrm{mc}\right.$ or $\left.{ }^{111} \mathrm{In}\right)$. 


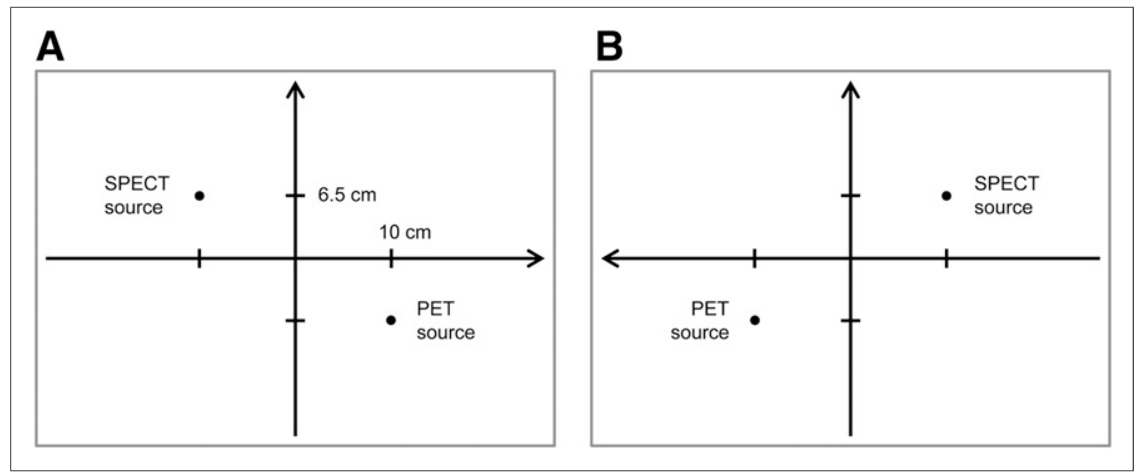

FIGURE 2. Positions of SPECT source (99mTc or $\left.{ }^{111} \mathrm{In}\right)$ and PET source $\left({ }^{68} \mathrm{Ga}\right)$ in images. (A) Relative to center position of close (lower) detector, SPECT source was positioned at coordinates $(-10 \mathrm{~cm},+6.5$ $\mathrm{cm})$, and SPECT source was positioned at symmetric coordinates $(+10 \mathrm{~cm},-6.5$ $\mathrm{cm})$. This gives distance of $24 \mathrm{~cm}$ between the 2 sources. (B) Seen from distant (upper) detector, positions are mirrored around vertical axis.

where the activity was calculated from the originally measured activity with decay correction until halfway into frame 4 (PET source, ${ }^{68} \mathrm{Ga}$ ) or frame 5 (SPECT source, ${ }^{99 \mathrm{~m}} \mathrm{Tc}$ or ${ }^{111} \mathrm{In}$ ).

To compare the sensitivity of a given area with PET and SPECT activity, the following ratio was calculated:

PET $/$ SPECT sensitivity ratio $=\frac{\text { sensitivity for PET source }}{\text { sensitivivity for SPECT source }}$

Eq. 5

Because "sensitivity for PET source" measures down scatter into the SPECT energy window or windows, a low PET/SPECT sensitivity ratio is preferable.

We did not measure both activities in the same location, because we would not be able to distinguish between downscattered photons from the PET source and photons originating from the SPECT source. Instead, a "same location" PET/SPECT sensitivity ratio was calculated by dividing the sensitivity within the PET ROI by the sensitivity within the SPECT ROI (i.e., Eq. 5, but with numerator and denominator from different ROIs). This mimics the presence of 2 sources in the same location at the same time.

\section{RESULTS}

\section{Energy Spectra}

Measured energy spectra for the PET source alone are shown in Figure 3. As expected, the measured energy spectrum from the PET source overlapped with the energy windows of the SPECT sources, and thicker septa $(\mathrm{HE}>$ MELP $>$ LEHR) reduced the number of photons detected from the PET source, both as $511-\mathrm{keV}$ photons and as scattered photons.

Spectra with and without scatter medium are shown in Figure 4. The down-scatter spectrum is approximately a factor of 2 lower with scatter medium than without scatter medium. Measurements with ${ }^{99 m}$ Tc over the same scatter phantom (spectra not shown) had down scatter below the ${ }^{99 \mathrm{~m}}$ Tc energy window and about 3 times fewer counts within the energy window.

\section{Dead Time, Counting Rates, and Sensitivities}

Dead times are reported in Table 2 as percentages. Especially for the LEHR collimator (measurement series A) and the close detector, the PET source resulted in considerable dead time.
The effect of dead time is seen in Figure 5A as a sudden drop in counting rate of ${ }^{99 \mathrm{~m}} \mathrm{Tc}$ (as measured within the SPECT ROI) when the PET source is introduced. With the MELP collimator, the PET source had a much lower effect on the counting rate (Fig. 5B). With the HE collimator, almost no effect of dead time was seen with the given source strengths (Fig. 5C).

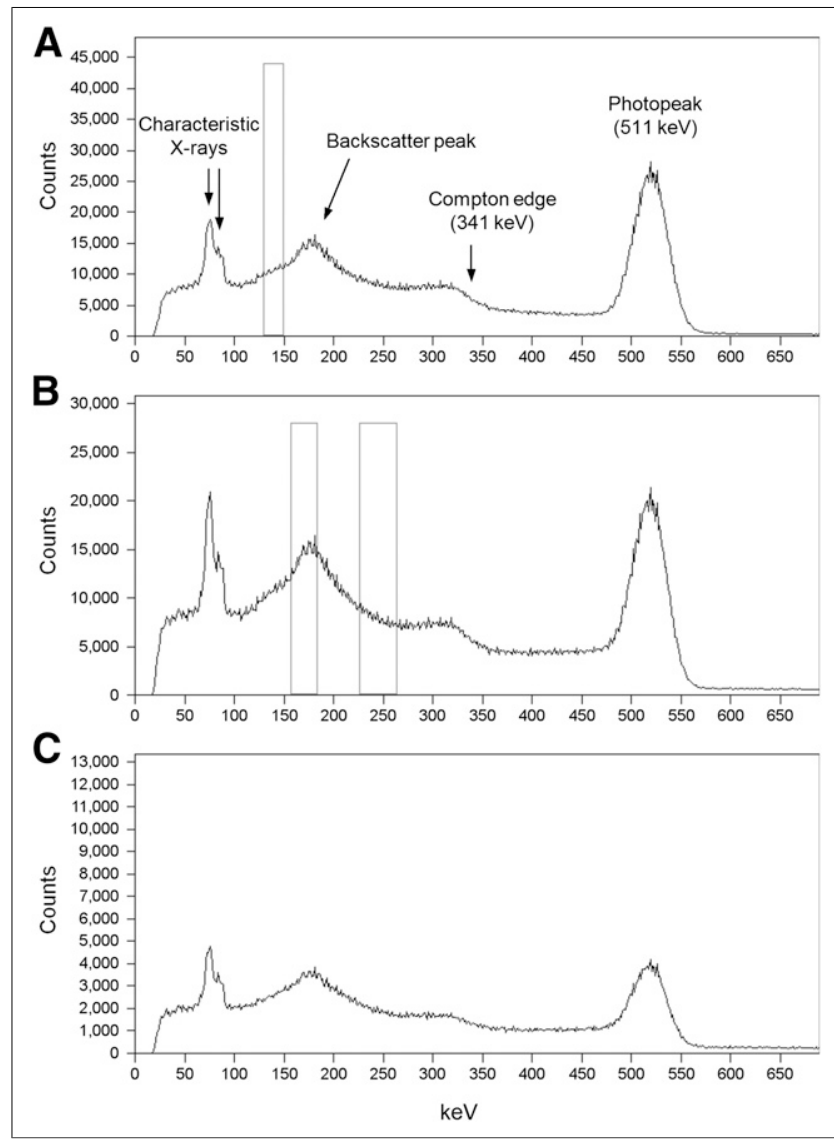

FIGURE 3. Energy spectrum from PET radionuclide measured with LEHR collimator (A), MELP collimator (B), and HE collimator $(C)$. Recording time was 10 times longer for MELP and HE collimators than for LEHR collimator. Note differences in vertical scale. Energy window for ${ }^{99 \mathrm{~m} T c}$ is shown on LEHR spectrum (A), and energy windows for ${ }^{111}$ In are shown on MELP spectrum (B). 


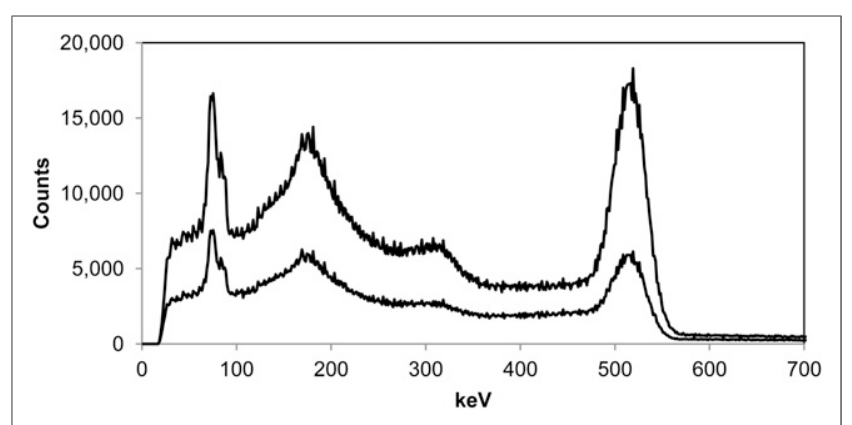

FIGURE 4. Comparison of measured energy spectra from PET activity without scatter medium (top curve) and with scatter medium (bottom curve).

In Figure 5D, the 3 collimators are compared by converting the curves in Figures $5 \mathrm{~A}-5 \mathrm{C}$ to PET/SPECT sensitivity ratios (Eq. 5).

Details on the sensitivities from measurement series A (LEHR collimator) are given in Table 3. As expected for this collimator, the PET source could give counts within the SPECT ROI even though it was in another position, whereas the SPECT source gave negligible counts within the PET ROI. The PET/SPECT sensitivity ratios (Eq. 5) and the sensitivities to the SPECT source are summarized for all measurement series in Table 4.

\section{Imaging with the PET Source Present}

LEHR Collimator (Measurement Series A). Figure 6 shows imaging of both the PET source and the SPECT source using the LEHR collimator. In the gray scale normalization suitable for the SPECT source, the PET source cannot be seen. To visualize the PET source, the gray scale was changed to $100 \%$ (black) at the position of the PET source (Fig. 7A). The LEHR collimator was unable to collimate the PET source. In this normalization, the image from the distant-detector (not shown) counts from the
PET source were seen over the entire detector as a weak gray background.

MELP Collimator (Measurement Series B and D). Like the LEHR collimator, the MELP collimator did not allow the PET source to be seen in images normalized to the SPECT source (images not shown). Normalizing gray scale to the PET source revealed that the MELP collimator at least partially collimated the PET source (Fig. 7B). The star artifact corresponds to the directions at which the photons penetrating the septa will encounter the least lead (compare the star pattern with the collimator top view in Fig. 1). The images with ${ }^{111}$ In as the SPECT source were similar and are not shown.

HE Collimator (Measurement Series $C$ and $E$ ). With the HE collimator, the effectiveness of collimation was increased further and the star artifact was reduced (Fig. 7C).

\section{DISCUSSION}

\section{Earlier Works}

PET radionuclides have been used with $\gamma$ cameras for 511-keV SPECT using UHE collimators. Additionally, cardiac dual-isotope SPECT imaging involving a PET radionuclide has been described in several papers (8-12). Two of these studies $(8,12)$ included a comparison of ${ }^{99 \mathrm{~m} T c-t r a c e r}$ imaging with and without the presence of $511 \mathrm{keV}$ by scanning the same subjects for ${ }^{99 \mathrm{~m}} \mathrm{Tc}$-sestamibi/tetrofosmin twice, before and after ${ }^{18} \mathrm{~F}-\mathrm{FDG}$ injection. The study by Delbeke et al. (8) did not find any clinically important differences, but their comparison involved only 4 patients. Pagnanelli et al. (12), who performed gated acquisitions and had 13 patients in their comparison study, found that perfusion defects of ${ }^{99 m} \mathrm{Tc}$-tetrofosmin were reduced (i.e., harder to see) in the dual-isotope scanning.

In the preclinical world, works on combined PET/SPECT scanners have been described in conference proceedings
FIGURE 5. (A-C) Counting rate per megabecquerel (sensitivity) for $99 \mathrm{mTc}$ SPECT source $(\bullet)$ and for PET source (O) for LEHR (A), MELP (B), and HE (C) collimators. To show effect of decay, calculation of $\mathrm{cps} / \mathrm{MBq}$ used activities fixed to time of first measurements (Table 2). The first points are single sources (PET or SPECT), and the following points are measured with both sources. Full curves are fitted to late data, that is, at a time when dead time is low. (D) PET/SPECT sensitivity ratio (Eq. 5) from these data for LEHR (dashed line), MELP (solid line), and HE (dotted line).

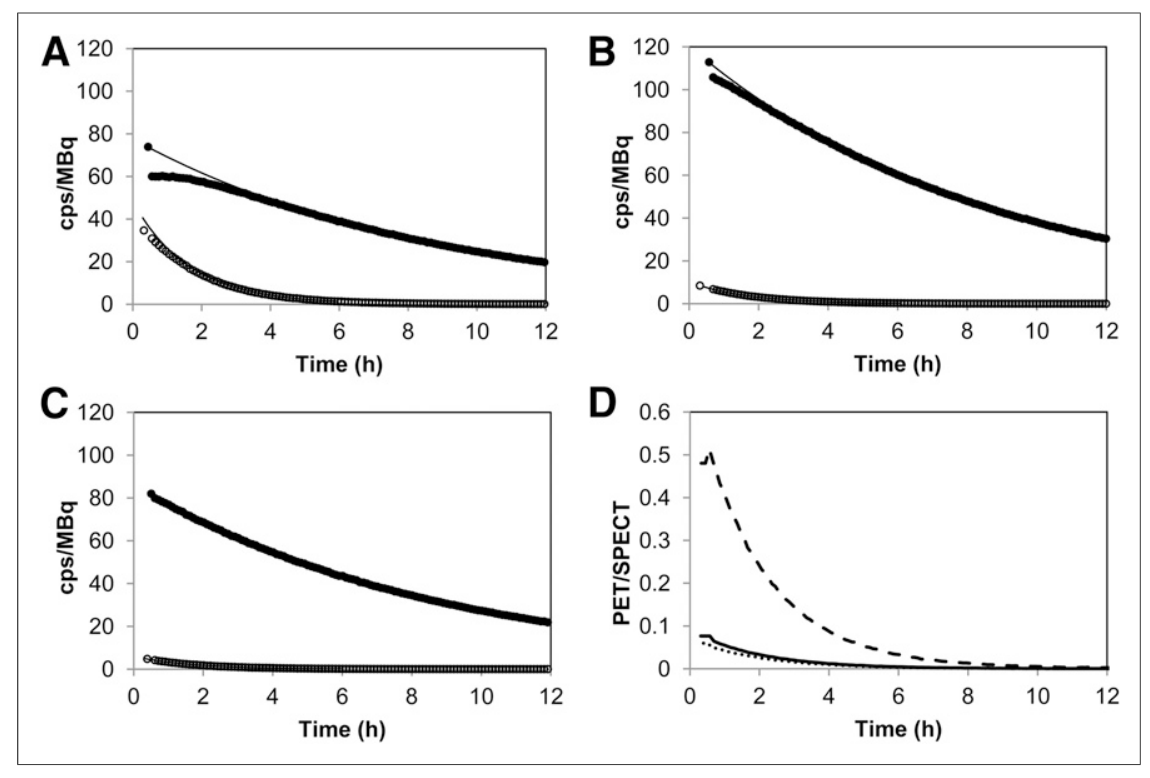


TABLE 3

Detailed Sensitivity Data for Measurement with LEHR Collimator

\begin{tabular}{|c|c|c|}
\hline \multirow[b]{2}{*}{$\begin{array}{l}\text { LEHR collimator, } \\
{ }^{68} \mathrm{Ga} \text { and } 99 \mathrm{mTc}\end{array}$} & \multicolumn{2}{|c|}{$\mathrm{cps} / \mathrm{MBq}$} \\
\hline & $\begin{array}{l}\text { Close } \\
(10 \mathrm{~cm})\end{array}$ & $\begin{array}{l}\text { Distant } \\
(\sim 60 \mathrm{~cm})\end{array}$ \\
\hline \multicolumn{3}{|l|}{ With PET source (frame 4) } \\
\hline Full detector & 2551 & 497 \\
\hline Within PET ROI & 36 & 2.6 \\
\hline Within SPECT ROI & 5.2 & 2.0 \\
\hline \multicolumn{3}{|c|}{ With SPECT source (frame 5) } \\
\hline Full detector & 82 & 73 \\
\hline Within SPECT ROI & 74 & 36 \\
\hline Within PET ROI & 0.001 & 0.006 \\
\hline \multicolumn{3}{|c|}{$\begin{array}{l}\text { cps }=\text { counts per second. } \\
\text { Data were calculated using Equation } 4 \text {. Figure } 6 \text { shows ROI } \\
\text { positions. If dead-time correction had been applied, numbers with } \\
\text { PET source would have been somewhat higher for close detector } \\
\text { and slightly higher for distant detector (Table 2). }\end{array}$} \\
\hline
\end{tabular}

(13-15) and are emerging in peer-reviewed literature $(5,16)$. Septal penetration and down scatter to the SPECT energy window are major concerns, as exemplified by the study by Chapman et al. (5) described at the start of this article.

Regarding standard $\gamma$ cameras equipped with standard collimators (i.e., non-UHE collimators), we have not been able to find any published studies that systematically investigated the effect of $511-\mathrm{keV}$ photons on low-energy imaging. In a broader scope, several papers have reported on the choice of collimator with ${ }^{123} \mathrm{I}$ (17-20), which has a main peak at $159 \mathrm{keV}$ but a small percentage of $\gamma$ emissions at higher energies. However, ${ }^{123} \mathrm{I}$ has a fixed percentage of high-energy photons to low-energy photons, whereas the combination of PET and SPECT radionuclides can involve both higher and lower ratios as well as other energies of the SPECT radionuclide photons.

\section{Present Study}

In the present study, we focused on using a standard $\gamma$ camera and collimators typically available for clinical studies. The most common PET radionuclide in clinical use is ${ }^{18} \mathrm{~F}$, but we chose to use ${ }^{68} \mathrm{Ga}$ because of its relatively short half-life $\left(\mathrm{T}_{1 / 2}=67.7 \mathrm{~min}\right)$, which allowed us to perform measurements until a time when almost no PET activity remained, simply by acquiring data overnight. Although PET radionuclides differ in the energy of the emitted positrons, influencing resolution $(21)$, the $511-\mathrm{keV}$ photons are common to all PET radionuclides.

\section{Down Scatter of $\mathbf{5 1 1} \mathrm{keV}$ to SPECT Radionuclide Energies}

Regardless of the type of collimator used and whether scatter medium is present, our measured energy spectra from the 511-keV (PET) source showed a photopeak at $511 \mathrm{keV}$, characteristic x-rays from lead below $100 \mathrm{keV}$, and a continuous spectrum in between (Figs. 3 and 4). Although the 511$\mathrm{keV}$ photopeak and the $\mathrm{x}$-rays are discriminated by the energy windows, the continuous spectrum results in counts that seemingly (but not truly) arise from the SPECT source.

TABLE 4

PET/SPECT Sensitivity Ratios

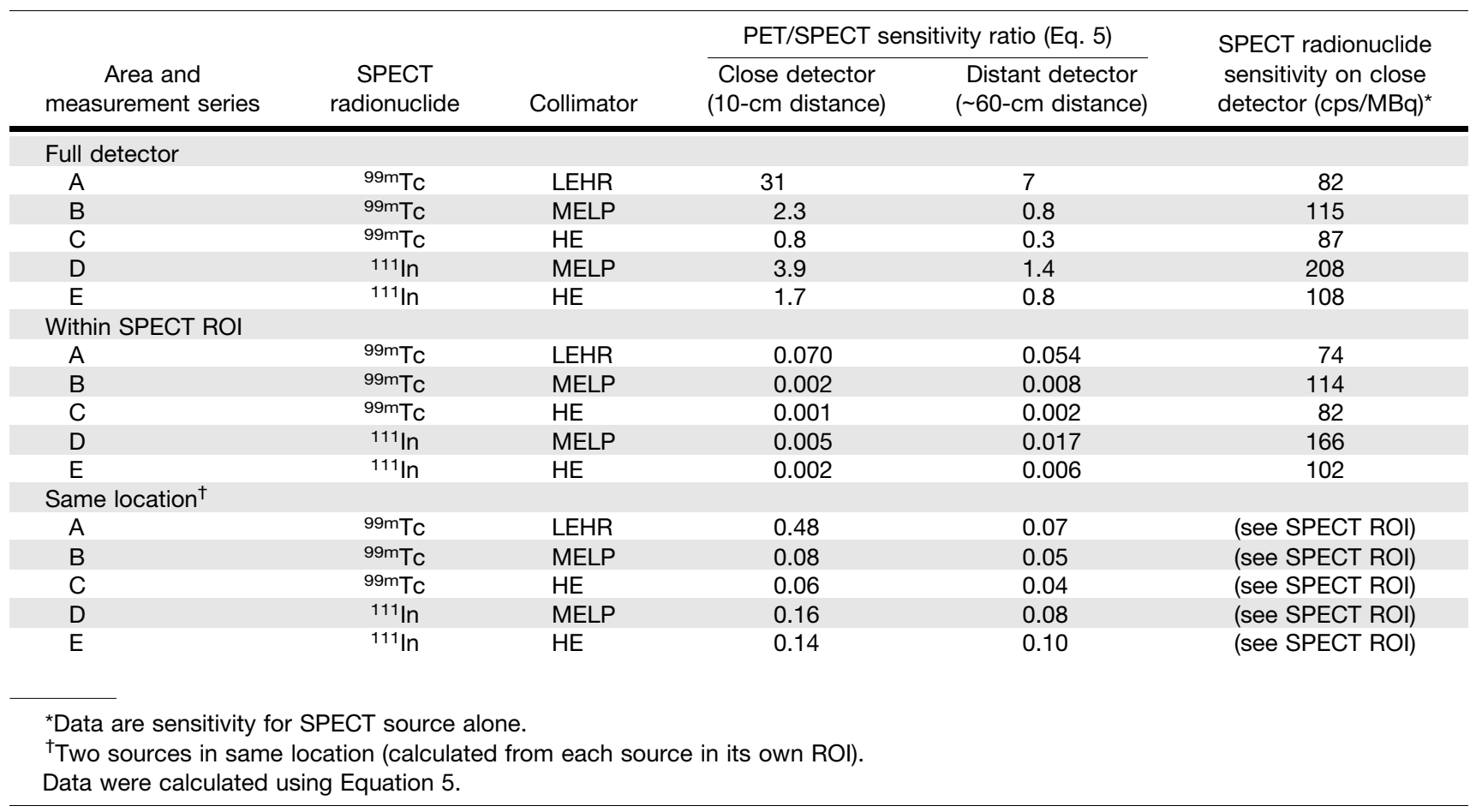


FIGURE 6. Images for LEHR collimator with ${ }^{99 m T c}$ as SPECT source and ${ }^{68} \mathrm{Ga}$ as PET source, with ROls drawn around positions of sources. On close detector (A), SPECT source is seen as spot, whereas on distant detector $(B)$, image of SPECT source is blurred because of distance. With the shown normalizing of the gray scale, PET source cannot be seen in images (Fig. 7 presents image A with a differently normalized gray scale).

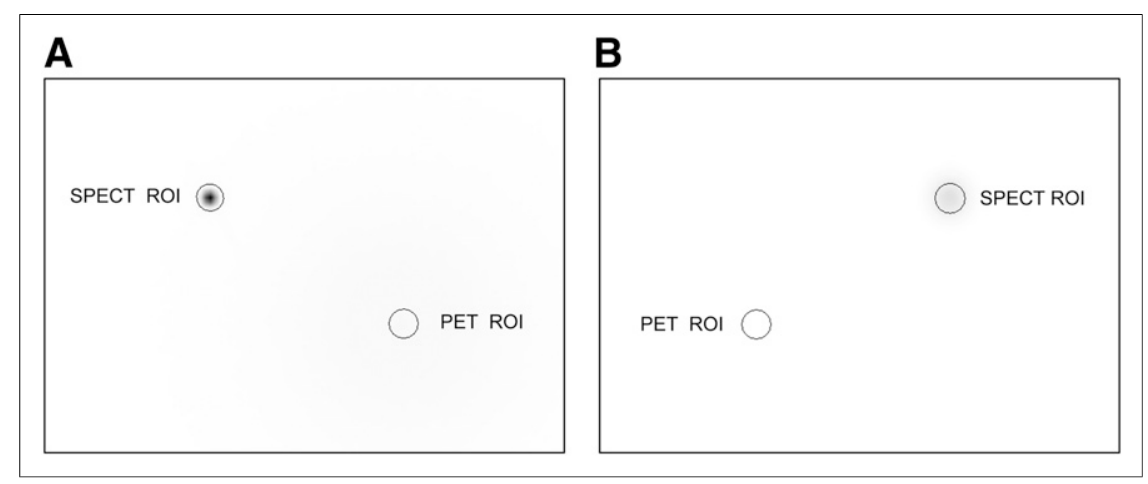

The continuous spectrum up to $341 \mathrm{keV}$ (the Compton edge) in Figure 3 results from single-scatter events, that is, 511-keV photons being scattered in the detector crystal and then escaping further detection. Alternatively, scattering can take place outside the crystal, followed by detection of the scattered photon. A 511-keV photon scattered once will have an energy from 170 to $511 \mathrm{keV}$. This energy will be lowest if the photon is scattered back in the direction from which it came. As such, the backscatter peak in Figure 3 corresponds to $511-\mathrm{keV}$ photons passing through the crystal without interaction, backscattering on the inside of the detector shielding, and then being detected in the crystal.

As seen in Figure 4, the introduction of a scatter medium both enhanced and attenuated the amount of scattered radiation: relative to the photopeak, scattered radiation was enhanced, but on an absolute scale it was attenuated by a factor of approximately 2 . The shape of the down scatter spectrum in the figure is very much the same as that without the scatter medium.

For ${ }^{99 \mathrm{~m}} \mathrm{Tc}$, the attenuation factor from the scatter medium was about 3 . Together with an attenuation factor of 2 for down scatter from $511 \mathrm{keV}$, this indicates that the PET/SPECT ratios would be about $50 \%$ higher with scatter medium than without scatter medium - that is, somewhat higher but of unchanged magnitude.

Down scatter can somewhat be compensated for if scatter windows are used to estimate the amount of scatter.
However, choosing the best scatter windows and compensation method is not simple (22). In the present study, we used a basic set-up without scatter windows. More complicated set-ups applying suitable scatter correction methods may achieve better results.

\section{LEHR Collimator}

The 511-keV photons from the PET source could largely penetrate the thin septa of the LEHR collimator, and a high dead time was registered from just a few megabecquerels of PET activity (Table 2). The high percentage of dead time turned out to be the most important issue when the LEHR collimator was used. In a preliminary study, we set out to use approximately $100 \mathrm{MBq}$ of PET activity because this is common for a patient immediately after a PET scan; but with this activity the $\gamma$ camera was saturated and unable to measure any counts on the closest detector. The effect of dead time from a more moderate PET activity is clearly seen in Figure 5A: the counting rate measured within the SPECT ROI has a sudden drop when $3.5 \mathrm{MBq}$ of PET activity are introduced.

A possible strategy could be to wait until the PET radionuclide has decayed to a suitably low level (somewhere below $1 \mathrm{MBq}$ ). But as illustrated by Figure 5D, the use of MELP or HE collimators can immediately reduce the influence of the PET radionuclide by a factor corresponding to several half-lives of waiting.

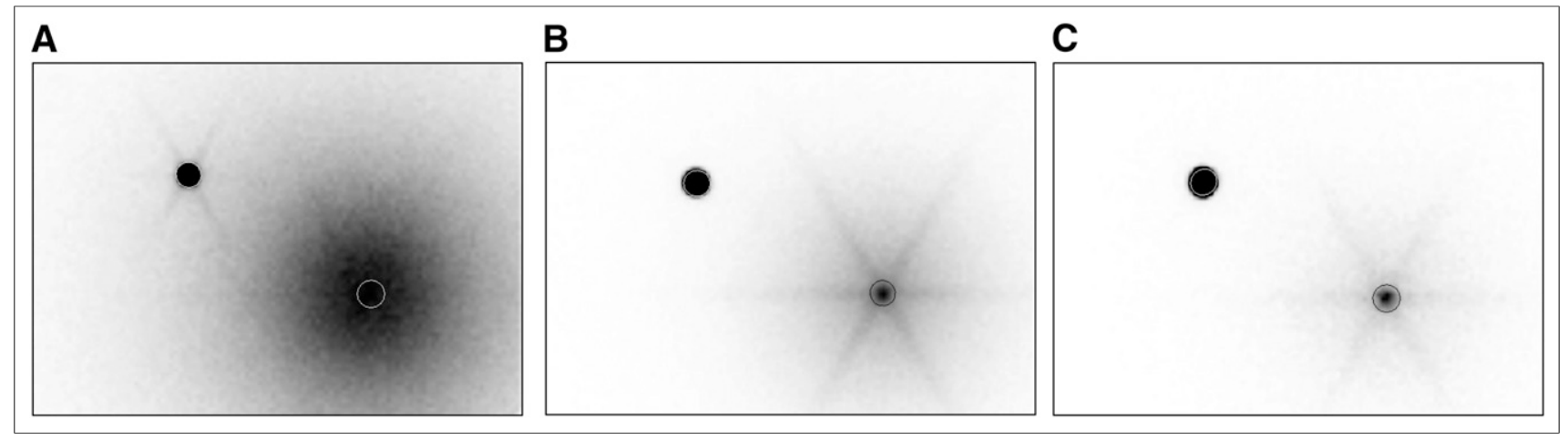

FIGURE 7. (A) Same image as in Fig. 6A (LEHR collimator, close detector) but normalized to maximal counting rate from PET source. (B) Corresponding image with MELP collimator. (C) Corresponding image with HE collimator. Images with ${ }^{111}$ In as SPECT radionuclide were similar to those with ${ }^{99 \mathrm{~m} T c}$ and are not shown. 


\section{MELP Collimator}

Compared with the LEHR collimator, the thicker septa of the MELP collimator reduced both the dead time and the PET/SPECT sensitivity ratios (Eq. 5), as seen by comparing measurement series A and B in Tables 2 and 4 . The cost of this reduction is poorer resolution, as indicated by the geometric resolution reported in Table 1 . On the positive side, the MELP collimator has favorable sensitivity for the SPECT isotopes, as seen in Table 4.

The PET/SPECT sensitivity ratio within a localized position was reasonably low with the MELP collimator. Within the same location (Table 4), the sensitivity for the PET source was only $5 \%-10 \%$ of that for ${ }^{99 \mathrm{~m}} \mathrm{Tc}$ and only $10 \%-20 \%$ of that for ${ }^{111} \mathrm{In}$. Remembering that scatter may increase the PET/SPECT sensitivity ratio somewhat, we use the upper numbers as estimates; that is, $10 \mathrm{MBq}$ of PET activity will give counts corresponding to about $1 \mathrm{MBq}$ of ${ }^{99 \mathrm{~m}} \mathrm{Tc}$ or about $2 \mathrm{MBq}$ of ${ }^{111} \mathrm{In}$.

If the PET activity is close to the detector, counts from this activity can be somewhat localized (Fig. 7), but with the low sensitivity, localized foci from the PET activity are unlikely to be seen on a color scale suitable for the SPECT foci, with the possible exception being a high and specific uptake of the PET tracer. Thus, in general, counts from residual PET activity will contribute mainly to the background in the images.

Collimator design can vary from one manufacturer to another, but taking the MELP collimator studied here as representative, we estimate that if a medium-energy collimator is used, up to $10 \mathrm{MBq}$ of PET activity can be tolerated for most SPECT imaging applications. According to our results, $10 \mathrm{MBq}$ of PET activity will at most give 5\% dead time; if activity is distributed in a body, lower dead time can be expected, partly because photons will be attenuated within the body and partly because only a fraction of the activity will be as close as $10 \mathrm{~cm}$ to the nearest detector.

\section{HE Collimator}

The high-energy collimator has thicker lead septa than the MELP and LEHR collimators. For the full detector, this reduces the PET/SPECT sensitivity ratio (Eq. 5) by a factor of 2-3 compared with the MELP collimator (Table 4, full detector: $\mathrm{C}$ vs. $\mathrm{B}$ and $\mathrm{E}$ vs. D).

Comparing sensitivities at the same location, we-somewhat surprisingly - found that the PET/SPECT sensitivity ratio for the HE collimator was not convincingly better than that for the MELP collimator (Table 4: C vs. B and E vs. D). This finding seems to be due to the fact that an HE collimator reduces not only septal penetration but also the sensitivity to the SPECT source (rightmost column in Table 4) because the thick septa have the side effect of causing a large part of the collimator surface to be lead rather than holes (Fig. 1). Thus, both wanted and unwanted photons are attenuated, and in localized positions, these 2 effects seem to cancel in the present context.
However, the HE collimator does favorably reduce the dead time (Table 2). Likewise, in Figure 7C only a minor star artifact is seen. However, the fact that the structure of the collimator is perceptible in the image can in itself result in noise or poorer resolution.

All in all, although the HE collimator is warranted for imaging of radionuclides that emit high-energy photons, such as ${ }^{131} \mathrm{I}$, its advantage over the MELP collimator is not convincing when only a few megabecquerels of PET activity is left in a patient who has been injected with many megabecquerels of a SPECT radionuclide.

\section{CONCLUSION}

The 511-keV photons originating from the PET radionuclides easily penetrate a low-energy collimator on a $\gamma$ camera, generating the risk of flooding the camera if the patient contains tens or hundreds of megabecquerels of PET activity, and even a few megabecquerels of PET activity may result in considerable dead time.

If medium-energy (ME) or high-energy (HE) collimators are available, dead time and cross-talk from PET activity will be far lower, and typically up to $10 \mathrm{MBq}$ of PET activity will be tolerable with ME collimators. The detectors will have a low sensitivity to PET activity compared with the SPECT activity, and foci from PET activity are unlikely to be seen. Ten megabecquerels of PET activity will result in counts corresponding to about $1 \mathrm{MBq}$ of ${ }^{99 \mathrm{~m}} \mathrm{Tc}$ or about $2 \mathrm{MBq}$ of ${ }^{111} \mathrm{In}$, and these counts will usually be seen as a diffuse background rather than as foci.

Unless high PET activity ( $>10 \mathrm{MBq})$ in the patient cannot be avoided, ME collimators seem preferable to HE collimators, partly because of their sensitivity and partly because the structure of the thick septa of HE collimators can be seen in planar imaging and may consequently increase the noise in tomographic imaging.

\section{DISCLOSURE}

No potential conflict of interest relevant to this article was reported.

\section{REFERENCES}

1. Rahmim A, Zaidi H. PET versus SPECT: strengths, limitations and challenges. Nucl Med Commun. 2008;29:193-207.

2. El Fakhri G. Ready for prime time? Dual tracer PET and SPECT imaging. Am J Nucl Med Mol Imaging. 2012;2:415-417.

3. Palestro CJ, Roumanas P, Swyer AJ, Kim CK, Goldsmith SJ. Diagnosis of musculoskeletal infection using combined In-111 labeled leukocyte and Tc99m SC marrow imaging. Clin Nucl Med. 1992;17:269-273.

4. Palestro CJ, Brown ML, Forstrom LA, et al. Society of Nuclear Medicine procedure guideline for ${ }^{111} \mathrm{In}$-leukocyte scintigraphy for suspected infection/ inflammation, version 3.0. Society of Nuclear Medicine and Molecular Imaging Web site. http://interactive.snm.org/docs/Leukocyte_v3.pdf. Published June 2, 2004. Accessed January 7, 2014.

5. Chapman SE, Diener JM, Sasser TA, et al. Dual tracer imaging of SPECT and PET probes in living mice using a sequential protocol. Am J Nucl Med Mol Imaging. 2012;2:405-414. 
6. Anger HO. Scintillation camera with multichannel collimators. J Nucl Med. 1964;5:515-531.

7. Formiconi AR. Collimators. Q J Nucl Med. 2002;46:8-15.

8. Delbeke D, Videlefsky S, Patton JA, et al. Rest myocardial perfusion/metabolism imaging using simultaneous dual-isotope acquisition SPECT with technetium99m-MIBI/fluorine-18-FDG. J Nucl Med. 1995;36:2110-2119.

9. Matsunari I, Kanayama S, Yoneyama T, et al. Electrocardiographic-gated dualisotope simultaneous acquisition SPECT using ${ }^{18} \mathrm{~F}-\mathrm{FDG}$ and ${ }^{99 \mathrm{~m}} \mathrm{Tc}$-sestamibi to assess myocardial viability and function in a single study. Eur J Nucl Med Mol Imaging. 2005;32:195-202.

10. Matsunari I, Kanayama S, Yoneyama T, et al. Myocardial distribution of ${ }^{18} \mathrm{~F}-$ FDG and ${ }^{99 m}$ Tc-sestamibi on dual-isotope simultaneous acquisition SPET compared with PET. Eur J Nucl Med Mol Imaging. 2002;29:1357-1364.

11. Sandler MP, Videlefsky S, Delbeke D, et al. Evaluation of myocardial ischemia using a rest metabolism/stress perfusion protocol with fluorine-18 deoxyglucose/ technetium-99m MIBI and dual-isotope simultaneous-acquisition single-photon emission computed tomography. J Am Coll Cardiol. 1995;26:870-878.

12. Pagnanelli RA, Hanson MW, Turkington T, Coleman RE, Borges-Neto S. Gated ${ }^{99 \mathrm{~m} T c-t e t r o f o s m i n}$ and ${ }^{18} \mathrm{~F}-\mathrm{FDG}$ studies: a comparison of single-acquisition and separate-acquisition protocols. J Nucl Med Technol. 2002;30:175-178.

13. Ma T, Shao Y, Yao R, Manchiraju P. PET and SPECT dual-tracer imaging: correction of $511 \mathrm{keV}$ photon's down-scatter effect on SPECT. In: Nuclear Science Symposium Conference Record. Piscataway, NJ: IEEE; 2007:3404-3407.

14. Shao Y, Yao R, Ma T, Manchiraju P. Initial studies of PET-SPECT dual-tracer imaging. In: Nuclear Science Symposium Conference Record. Piscataway, NJ: IEEE; 2007:4198-4204.
15. Bartoli A, Belcari N, Del Guerra A, Fabbri S. Simultaneous PET/SPECT imaging with the small animal scanner YAP-(S)PET. In: Nuclear Science Symposium Conference Record. Piscataway, NJ: IEEE; 2007:3408-3413.

16. Yao R, Deng X, Beaudoin JF, et al. Initial evaluation of LabPET/SPECT dual modality animal imaging system. IEEE Trans Nucl Sci. 2013;60:76-81.

17. Bolmsjö MS, Persson BR, Strand SE. Imaging ${ }^{123}$ I with a scintillation camera: a study of detection performance and quality factor concepts. Phys Med Biol. 1977;22:266-277.

18. De Geeter F, Franken PR, Defrise M, Andries H, Saelens E, Bossuyt A. Optimal collimator choice for sequential iodine-123 and technetium-99m imaging. Eur J Nucl Med. 1996;23:768-774.

19. Inoue Y, Shirouzu I, Machida T, et al. Physical characteristics of low and medium energy collimators for ${ }^{123} \mathrm{I}$ imaging and simultaneous dual-isotope imaging. Nucl Med Commun. 2003;24:1195-1202.

20. Snay ER, Treves ST, Fahey FH. Improved quality of pediatric ${ }^{123}$ I-MIBG images with medium-energy collimators. J Nucl Med Technol. 2011;39:100104.

21. Jødal L, Le Loirec C, Champion C. Positron range in PET imaging: an alternative approach for assessing and correcting the blurring. Phys Med Biol. 2012;57:3931-3943.

22. Lagerburg V, de Nijs R, Holm S, Svarer C. A comparison of different energy window subtraction methods to correct for scatter and downscatter in I-123 SPECT imaging. Nucl Med Commun. 2012;33:708-718.

23. Symbia $S$ and $T$ system specifications. Siemens Web site. http://www.medical. siemens.com/siemens/en_GB/gg_nm_FBAs/files/brochures/2007/SymbiaST_spec_ sheet.pdf. Published 2007. Accessed January 7, 2014. 